\title{
RPUDD
}

畨

Revista Pedagogía Universitaria y Didáctica del Derecho

Primer Semestre, año 2017.

Volumen 4, número 1.

La Revista Pedagogía Universitaria y Didáctica del Derecho es una publicación de la Unidad de Pedagogía Universitaria y Didáctica del Derecho, de la Facultad de Derecho de la Universidad de Chile. Es una publicación internacional, con trabajo conjunto entre Chile y Brasil. La Revista tiene por objetivo central configurarse como un espacio académico de encuentro entre investigadores, abogados y expertos en educación (licenciados en educación, profesores, psicólogos educacionales y sociólogos de la educación) a propósito de la investigación sobre pedagogía universitaria, docentes universitarios, estudiantes universitarios, enseñanza-aprendizaje del derecho, prácticas docentes, profesión jurídica y currículo.

Revista Pedagogía Universitaria y Didáctica del Derecho En línea. Coodirección

Chile Dra. María Francisca Elgueta. Brasil Dr. Renato Duro Dias. ISSN 0719-5885

rpedagogia@derecho.uchile.cl +5629785397

Algunos derechos reservados. Publicada bajo los términos de la licencia Creative Commons atribución - compartir igual 4.0 internacional.

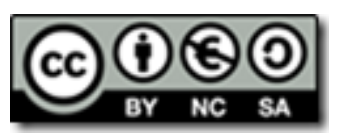




\section{EL USO DE LOS ENTORNOS PERSONALES DE APRENDIZAJ E (PLE) EN LOS CURSOS DE INTRODUCCIÓN AL DERECHO}

\section{Use of Personal Learning Environment in Introduction of Law \\ courses}

Katherine Becerra Valdivia ${ }^{1}$

Resumen: Hoy estamos inmersos en una sociedad que utiliza las TIC para muchas de las actividades diarias, y la educación no ha estado ajena a estos cambios. De hecho, actualmente se habla de tecnologías del aprendizaje y del conocimiento (TAC), por la gran importancia que han ido adquiriendo en el proceso de enseñanza-aprendizaje. En este contexto existen los entornos personales de aprendizaje (PLE) que permiten a los estudiantes realizar un trabajo autónomo de adquisición de conocimientos y donde el profesor es un facilitador. En este trabajo se presenta una experiencia de innovación utilizando PLE en el curso de introducción al Derecho.

Palabras Claves: TIC, Tecnologías del Aprendizaje y del Conocimiento, Entornos personales de aprendizaje.

Abstract: Today we live inside of a society which use ICT for many daily activities, and education is not been outside of those changes. Actually, we talk

1 Abogada. Licenciada en Ciencias Jurídicas y Magister en Derecho por la Universidad Católica del Norte, Académica de Derecho Constitucional e Introducción al Derecho de la Facultad de Ciencias J urídicas, Escuela de Derecho, Coquimbo, de la misma casa de estudio. Actualmente estudiante del Magíster en Pedagogía Universitaria de la Universidad Mayor, Santiago, Chile. Dirección: Larrondo 1281, Coquimbo, Chile. E-mail: kbecerra@ucn.cl

DOI: 10.5354/0719-5885.2017.46255 
about knowledge and learning technologies, for the great importance of them in the teaching-learning process. In this context exist the personal learning environment (PLE) that permit to the student to work with autonomy to gain knowledge and the professor is a facilitator of this process. This article presents an experience of innovation using PLE in the Introduction of Law course.

Key words: ICT, Knowledge and learning technologies, Personal Learning Environment.

\section{Introducción}

Lograr que los estudiantes de la Escuela de Derecho de la Facultad de Ciencias J urídicas de la Universidad Católica del Norte, puedan utilizar una serie de tecnologías en la sala de clases no ha sido fácil, pues hay mucha reticencia en el ámbito de la disciplina en el uso de estas herramientas.

La realidad hoy supera dicho rechazo y hoy es posible encontrar las tecnologías de la información y la comunicación (TIC), las tecnologías del aprendizaje y conocimiento (TAC), e incluso las tecnologías del empoderamiento y participación (TEP). Por lo tanto, es esencial que en la sala de clases se utilicen como herramienta de apoyo al proceso de enseñanzaaprendizaje.

Las TAC se han vuelto indispensables en este proceso y a través de diversas técnicas y herramientas hoy se están integrando en la sala de clases. Una de ellas son los entornos personales de aprendizaje (PLE) que permiten desarrollar un trabajo autónomo por parte de los estudiantes, haciendo que el aprendizaje sea más flexible.

El trabajo que a continuación se presenta muestra una actividad en donde se utiliza el PLE, en un curso de Introducción al Derecho, y por tanto tendrá el siguiente plan de trabajo: se comenzará con la explicación conceptual de las TIC y TAC, así como el rol del profesor; luego se revisará que es el PLE, para seguir con el diseño de la actividad, teniendo claro el contexto y el programa del curso; finalizando con algunas conclusiones. 
REVISTA PEDAGOGÍA UNIVERSITARIA Y DIDÁCTICA DEL DERECHO [ISSN 0719-5885]

Unidad de Pedagogía Universitaria y Didáctica del Derecho, Universidad de Chile

$\mathrm{N}^{\circ}$ 1, vol. 4, $1^{\circ}$ semestre de 2017, 225-253 pp.

\section{Usando las TIC y las TAC2}

Es evidente que la sociedad ha sufrido acelerados cambios en las últimas décadas. En el entorno de las tecnologías los avances resaltan aún más, hoy vivimos en una cultura digital, caracterizada por una absorción tecnológica en todos los sectores de la sociedad, y la educación no es una excepción ${ }^{3}$. Hoy las TIC han sido esenciales para configurar un mundo mucho más conectado inmerso en la complejidad, y estas herramientas han dado paso a las denominadas TAC, las cuales están teniendo un rol esencial en la educación.

El Derecho si bien no ha estado ajeno a estos cambios, la introducción de estas tecnologías ha sido lenta, y muchas veces criticada en virtud de la denominación de enseñanza clásica del área4

\section{LAS TECNOLOGÍAS DE LA INFORMACIÓN Y LAS COMUNICACIONES}

Las TIC fueron incorporadas a lo largo de los años en diferentes programas de educación superior con el simple fin de que sus estudiantes lograran aprender un manejo óptimo de aparatos tecnológicos para facilitar su desempeño laboral en las diferentes áreas. La introducción de éstas en las

2Parte de este apartado fue realizado para un trabajo del Magister en Pedagogía Universitaria de la Universidad Mayor de la asignatura de "Tecnologías de la información y las Comunicaciones “, en conjunto con Katherine Etchart, Ángela Torreblanca y Karim Vega.

3 J ohn Granados Romero, “Las tecnologías de la información y las comunicaciones, las del aprendizaje y del conocimiento y las tecnologías para el empoderamiento y la participación como instrumentos de apoyo al docente de la universidad del siglo XXI", Medisur 12, n. ${ }^{\circ} 1$ (2014): 289-294; Santiago Nieto Martín y María J osé Rodríguez Conde, "Convergencia de resultados en dos diseños de investigación-innovación en enseñanza universitaria a través de las TIC", Revista Española de Pedagogía 65, n. 236 (2007): 27-48; Vicente Prieto et al. “Impacto de las tecnologías de la información y las comunicaciones en la educación y nuevos paradigmas del enfoque educativo", Revista Cubana de Educación Médica Superior 25. n. ¹ (2011): 95-102.

4 Christian Scheechler, “¿El buey y la carreta? Las TIC al servicio de la planificación de la enseñanza del Derecho", en Nuevos Paradigmas e innovaciones en la Enseñanza del Derecho: Actas II Seminario de Enseñanza del Derecho, coord. por Katherine Becerra (Santiago: Ediciones J urídicas de Santiago, 2015), 89. 
REVISTA PEDAGOGÍA UNIVERSITARIA Y DIDÁCTICA DEL DERECHO [ISSN 0719-5885]

Unidad de Pedagogía Universitaria y Didáctica del Derecho, Universidad de Chile

$\mathrm{N}^{\circ}$ 1, vol. 4, $1^{\circ}$ semestre de 2017, 225-253 pp.

Universidades del mundo ha sido uno de los principales factores de inducción al cambio y adaptación a las nuevas formas de hacer y de pensar 5 .

Para poder incorporarlas en nuestras aulas, se hace necesario identificar algunas de las principales características de las Tics y sus utilidades, las cuales según Mela ${ }^{6}$ son:

a. Inmaterialidad: Nos permite almacenar grandes cantidades de soportes en pequeños dispositivos.

b. Instantaneidad: Nos facilita la inmediatez, consiguiendo información aun encontrándonos alejados de la fuente.

c. Interactividad: Nos permite la comunicación bidireccional, entre personas o grupos.

d. Automatización de tareas: Gracias a esta característica podemos programar actividades que se realizan automáticamente con total seguridad y efectividad.

Por otra parte, las TIC propiamente tal, pueden clasificarse en variados tipos, según la misma autora:

a. Redes: Corresponden a la telefonía fija y móvil, banda ancha, red de televisión o redes en el hogar.

b. Terminales: Corresponden a ordenadores, navegador de internet, sistemas operativos para ordenadores, los teléfonos móviles, los televisores, reproductores portátiles de audio, videos o consolas de juego.

c. Servicios: Son lo que las TIC ofrecen a los consumidores como: correo electrónico, la e-sanidad, la educación, los videojuegos y los servicios móviles como los peer to peer PZP, los blogs o las comunicaciones

5 María López De La Madrid, “Usos de las TIC en la educación superior en México. Un caso de estudio", Revista Apertura (2007): 63-81; Rebeca Garzón Clemente, "Políticas públicas de inclusión de las tecnologías de la información y comunicación en la educación superior mexicana", Revista de Pedagogía 35 y 36, n. 97-98.

6 Marta Mela, ¿Qué son las TIC?, 2005 (consultado el 20 de mayo de 2015) http:/ / noticias.iberestudios.com/\%C2\%BFque-son-las-tic-y-para-que-sirven/ 
virtuales y escuelas de negocios que se especializan en impartir su información7.

Para referirnos a la apropiación de las TIC en educación debemos mencionar y analizar el término "Disonancia cognitiva"8. Éste se refiere a la tensión que se produce cuando existen contradicciones entre ideas, aspectos importantes de lo que se piensa, lo que vamos interiorizando y la realidad que se vive. Cuando este fenómeno se da en forma constante en nuestras vidas, produce malestar y nos lleva a generar nuevas ideas, actitudes y comportamientos para resolver este dilema.

Respecto a lo anterior, debemos reflexionar que lo que el estudiante desea es que haya una conexión entre el mundo extra online y su mundo real. Ellos necesitan una realidad más parecida a lo que viven en lo virtual, integración entre la web y la realidad, de acuerdo a su percepción completamente post digital y ausente de barreras entre ambas.

Las TIC facilitan la inducción al cambio y la adaptación de nuevas formas de pensar, así como el acceso a la información, generando un espacio de interactividad, que promueve la motivación y la eficiencia y mejora el conocimiento, en un entorno flexible ${ }^{9}$. En el fondo las TIC nos entregan herramientas que dicen relación con el cómo aprendemos dándonos diversas posibilidades en el aprendizaje.

Y esto último es lo que puede resultar más atractivo a los estudiantes: Hacer una universidad más flexible ${ }^{10}$, integrando las TIC, pero respetando las tradiciones e idiosincrasias de las carreras.

Dicho lo anterior, reafirmamos que las denominadas TIC, tecnologías de la información y las comunicaciones, hoy han venido a quedarse en nuestras vidas

\footnotetext{
${ }^{7}$ Mela, ¿Que son las TIC?

${ }^{8}$ León Festinger, A theory of cognitive dissonance (Evanston. Illinois: Row Peterson, 1957).

${ }^{9}$ López De La Madrid, “Usos de las TIC”.

${ }^{10} \mathrm{~J}$ esús Salinas, “Innovación docente y uso de las TIC en la enseñanza universitaria”. Revista Universidad y Sociedad del Conocimiento 1, n. ${ }^{\circ} 1$ (2004): 1-16.
} 
REVISTA PEDAGOGÍA UNIVERSITARIA Y DIDÁCTICA DEL DERECHO [ISSN 0719-5885]

Unidad de Pedagogía Universitaria y Didáctica del Derecho, Universidad de Chile

$\mathrm{N}^{\circ}$ 1, vol. 4, $1^{\circ}$ semestre de 2017, 225-253 pp.

diarias y las podemos utilizar de manera integrada en nuestras clases, dando paso a "la era de las TAC".

\section{TECNOLOGÍAS DEL APRENDIZAJ E Y EL CONOCIMIENTO}

Estas tecnologías se definen como "medios de enseñanza que, como tales, son elementos curriculares, que por sus sistemas simbólicos y estrategias de utilización propician el desarrollo de habilidades cognitivas en las personas, en un contexto determinado, facilitando y estimulando la intervención mediada sobre la realidad, la captación y la comprensión de la información por el estudiante y la creación de escenografías comunicativas diferenciadas que propician el aprendizaje y la interacción entre las personas que participan en el acto educativo."11.

Con esta definición queda de manifiesto que estas tecnologías tienen por objetivo facilitar el proceso de enseñanza aprendizaje, usando las herramientas que hoy por hoy, están disponibles para hacer más apropiado la construcción del conocimiento. En este sentido, hoy estamos dentro de una teoría de la educación que se denomina conectivismo, que "es la integración de principios explorados por las teorías de caos, redes, complejidad y autoorganización." ${ }^{12}$ donde lo esencial es que esta construcción del saber, se hace de manera colectiva e interrelacionada con los otros a través de los medios tecnológicos que existen.

Con el tiempo, la sociedad en sí ha presentado cambios y con estos se han generado avances y modificaciones en el uso de las tecnologías. Autores e investigadores se dan cuenta, que el concepto de TIC queda muy "pequeño" para los múltiples usos y beneficios que éstas pueden entregar, ya no sólo como una

${ }^{11}$ Julio Cabero, "Nueva mirada de las TIC aplicadas en la Educación Superior", Revista Digital Andalucía Educativa (2000): 1-14; Ángel Boza y Sara Conde, "Web 2.0 en educación superior: formación, actitud, uso, impacto, dificultades y herramientas", Digital Education Review, n. 28 (2015): 45-58.

${ }^{12}$ Georges Siemmens, "Conectivismo: Una teoría de aprendizaje para la era digital”, 2004, (consultado el 15 de octubre de 2015). www.diegoleal.org/docs/2007/Siemens(2004)Conectivismo.doc 
REVISTA PEDAGOGÍA UNIVERSITARIA Y DIDÁCTICA DEL DERECHO [ISSN 0719-5885]

Unidad de Pedagogía Universitaria y Didáctica del Derecho, Universidad de Chile

$\mathrm{N}^{\circ}$ 1, vol. 4, $1^{\circ}$ semestre de 2017, 225-253 pp.

simple herramienta de cómputo. Así surgió una nueva terminología denominada TAC.

Las TAC o tecnologías del aprendizaje y del conocimiento, pretenden ahondar más en la metodología y no quedarse sólo con el "saber usar", sino que pretenden que profesores y estudiantes exploren herramientas tecnológicas para facilitar el aprender en sí $^{13}$. Estas tecnologías son "un concepto creado por Vivancos, para explicar las nuevas posibilidades que las tecnologías abren a la educación, cuando éstas dejan de usarse como un elemento meramente instrumental cuyo objeto es hacer más eficiente el modelo educativo actual”14. Por lo tanto, inciden en la metodología, en los usos de la tecnología y no únicamente en dominar el uso de una serie de herramientas informáticas ${ }^{15}$.

Las TAC tratan de orientar las tecnologías de la información y la comunicación (TIC) hacia usos más formativos, tanto para el estudiante como para el profesor, con el objetivo de aprender más y mejor.

En la actualidad, todas las tecnologías han pasado a formar parte del quehacer diario y si bien, aún queda mucho camino por recorrer en las TAC, ya que se debe lograr que los docentes las incorporen y de buena forma, ya se ha dado paso a lo que se denomina TEP (tecnologías del empoderamiento y la participación) dónde nativos digitales ya las utilizan para llamar a la ciudadanía a participar y "hacerse escuchar", y esto también puede ocurrir en la sala de clases.

Las TAC se relacionan directamente con los entornos personales de aprendizajes o PLE.

13 "Tecnologías para el aprendizaje y el conocimiento", entrada publicada por Ramiro Aduviri, 2012, consultado el 19 de mayo de 2015. http:// es.slideshare.net/ ravsirius/ tecnologaspara-el-aprendizaje-y-el-conocimiento.

14, Paloma Recuero, “¿Qué es eso del TIC, TAP TEC?” 2013 (consultado el 19 de mayo de 2015). https:/ / palomarecuero.wordpress.com/ 2013/ 04/ 26/ que-es-eso-del-tic-tac-tep/

15 Granados et al., "Las tecnologías de la información”. 
REVISTA PEDAGOGÍA UNIVERSITARIA Y DIDÁCTICA DEL DERECHO [ISSN 0719-5885]

Unidad de Pedagogía Universitaria y Didáctica del Derecho, Universidad de Chile

$\mathrm{N}^{\circ}$ 1, vol. 4, $1^{\circ}$ semestre de 2017, 225-253 pp.

\section{ROL DEL PROFESOR EN ESTAS NUEVAS TECNOLOGÍAS}

Las TIC y TAC hoy han impactado en la forma como se hace docencia en nuestras instituciones de educación superior, y por tanto el rol del profesor ha cambiado sustancialmente, aun cuando encontramos muchas resistencias por parte de algunos docentes que niegan a este cambio socio-cultural.

Hoy el profesor más que una persona que entrega verdades absolutas es un facilitador de un proceso, un mediador ${ }^{16}$, y que al tratar de construir conocimiento es un aprendiz más cuando intenta aplicar TIC, pues generalmente los estudiantes saben más de estas tecnologías que los profesores.

Hoy más que hablar de las clásicas teorías del aprendizaje como el conductivismo, cognotivismo o constructivismo, estamos hablando de una nueva denominada conectivismo, según lo ya señalado, lo que se encuentra novedoso de esta visión es el entendimiento que el proceso de aprendizaje se basa no solo en lo interno del ser humano, sino que también en lo externo, uniendo diversos nodos de información, que se pueden ir adaptando al cambio.

Teniendo esto como premisa, me parece esencial revisar el concepto de desarrollo profesional docente, pues los profesores debemos ser parte de este flujo constante de readecuación al cambio. El desarrollo profesional docente es definido como un proceso de aprendizaje de los docentes en su labor profesional de enseñanza aprendizaje, para poder realizar de mejor manera sus clases y robustecer, a su vez, el proceso de enseñanza-aprendizaje de sus propios estudiantes ${ }^{17}$.

16 “El rol del maestro y del alumno en la web 2.0", entrada publicada por Henry Cárdenas, consultado el 5 de mayo de 2015. http:/ / henrycardenasrincon.blogspot.com/

17 Linda Castañeda y Jordi Adell, "El desarrollo profesional docente en los entornos personales de aprendizajes (PLE)", en Entornos personales de aprendizaje: claves para el ecosistema educativo en red, ed. por Linda Castañeda y J ordi Adell (Alicante: Marfil, 2011); Linda Castañeda y Jordi Adell, “Más allá de la tecnología: análisis de los entornos de aprendizaje personales y grupales de estudiantes en una asignatura universitaria". Cultura y Educación 26, n. 4 (2014): 739-774; Oskar Casquero et al., "Entornos de aprendizaje personales, educación superior y analítica del aprendizaje: un estudio sobre los efectos de la 
REVISTA PEDAGOGÍA UNIVERSITARIA Y DIDÁCTICA DEL DERECHO [ISSN 0719-5885]

Unidad de Pedagogía Universitaria y Didáctica del Derecho, Universidad de Chile

$\mathrm{N}^{\circ} 1$, vol. $4,1^{\circ}$ semestre de 2017, 225-253 pp.

Respecto a cómo introducir las TAC a las prácticas docentes, es esencial una palabra y que tiene que ver con el cambio: "flexibilidad", entendiendo que el profesor no maneja todo el contenido de la clase, y la misma ya no puede ser lineal, en el sentido de que se debe permitir los aportes de los estudiantes, gracias a la utilización de nuevas informaciones que se van adquiriendo, fomentando el pensamiento crítico ${ }^{18}$. Además, es esencial tener claro qué se quiere lograr con las TAC, cuál es el objetivo de aprendizaje y luego de eso ver cuáles son las herramientas más idóneas ${ }^{19}$.

La web 2.0, hoy nos permite introducir variados elementos que van desde utilizar correo electrónico para comunicarse con los estudiantes, que sería lo más usado, hasta ser capaces de crear con nuestros estudiantes nuevos conocimientos que ofrecer a la web.

Como profesores, todos hemos ido adquiriendo nuevos conocimientos disciplinares, y es un deber para el docente mantenerse actualizados en la/ las materia/as que impartimos; en los conocimientos pedagógicos para facilitar el proceso con los estudiantes y poder gatillar procesos de aprendizaje, y sin lugar a dudas también hoy es un deber poder integrar a las prácticas conocimientos tecnológicos, lo que sin duda es instrumental para el mejor desarrollo de nuestras clases, esto que es conocido como el modelo TPACK (Technological, Pedagogical and Content Knowledge), que implica saber utilizar una

multiplicidad de servicios en las redes personales de estudiantes universitarios", Cultura y Educación 26, n. 4 (2014): 696-738.

18 María Del Pilar Sepúlveda e Ignacio Calderón, “Las TICs y los procesos de enseñanzaaprendizaje: la supremacía de las programaciones, los procesos de enseñanza y las calificaciones ante las demandas de la sociedad del conocimiento", Revista Iberoamericana de Educación 45, n5 (2007); Carolina Cabrera Di Piramo y Amílcar Davyt, "Relaciones entre modelos de calidad de la educación superior y de políticas de ciencia, tecnología e innovación", Revista Iberoamericana de Educación Superior 8, n. 21 (2017): 109-122; Enrique Ruiz-Velasco Sánchez y Claudia Ortega Barba, "Tecnologías de la información y la comunicación para la innovación educativa”, Perfiles Educativos 36, n. 144 (2014): 214-218.

19 Isabel Brincones, "La incorporación de las TIC al proceso de enseñanza y aprendizaje". Revista de Investigación e Innovación Educativa del Instituto Universitario de Ciencias de la Educación, Universidad Autónoma de Madrid. 
metodología efectiva para el uso de las TIC y TAC apoyando estrategias y métodos pedagógicos en relación a una disciplina, surge para identificar los conocimientos requeridos para integrar las tecnologías en la docencia ${ }^{20}$ dentro de nuestras posibilidades, desde lo más fácil, hasta lo más complejo.

En este sentido también es importante agregar que los profesores deben tener ciertas competencias tecnológicas para poder hacer esta integración Las competencias son "un conjunto de comportamientos sociales, afectivos y habilidades cognoscitivas, psicológicas, sensoriales y motoras que permiten llevar a cabo adecuadamente un papel, un desempeño, una actividad o una tarea"21 y en nuestra sociedad del conocimiento es esencial que seamos capaces de poder recibir y transmitir la información en base a TIC $^{22}$ y por supuesto en base a TAC.

Este autor recién referenciado señala que "se hace necesario que los sujetos estén capacitados para movilizar y utilizar las nuevas herramientas de comunicación que tienen a su disposición en la sociedad del conocimiento, y ello pasa necesariamente por un nuevo tipo de alfabetización, que se centre no sólo en los medios impresos y sus códigos verbales, sino también en la diversidad de medios multimedia con que nos encontramos, y en los códigos icónicos que movilizan"23. Sería interesante describir cual es el mínimo competencial que se requiere para lograr con éxito la tarea, lo que por su extensión y profundizar no es objeto de este trabajo.

${ }^{20}$ De Benito et al. "Agregación, filtrado y curación para la actualización docente”, Píxel-Bit. Revista de Medios y Educación n. ${ }^{\circ} 42$ (2013): 157-169.

${ }^{21}$ Yolanda Argudín, Educación basada en competencias: nociones y antecedentes (México: Trillas, 2005), 15; Rafaela Herrero Martínez et al. "Formación centrada en competencias estudiantiles en Educación Superior", Revista de Ciencias Sociales 21, n. 4 (2015): 461-478; Ana Patricia León, "Estrategias de aprendizaje en educación superior en un modelo curricular por competencias", Revista de la Educación Superior 43, n. ${ }^{\circ} 172$ (2014): 123-144.

22 Julio Cabero, "Capacidades tecnológicas de las TICs por los estudiantes". Enseñanza \& Teaching: Revista interuniversitaria de didáctica n. ${ }^{\circ} 24$ (2006): 159-175.

${ }^{23}$ Cabero, "Capacidades tecnológicas". 
REVISTA PEDAGOGÍA UNIVERSITARIA Y DIDÁCTICA DEL DERECHO [ISSN 0719-5885]

Unidad de Pedagogía Universitaria y Didáctica del Derecho, Universidad de Chile

$\mathrm{N}^{\circ} 1$, vol. $4,1^{\circ}$ semestre de 2017, 225-253 pp.

\section{Los Entornos Personales de Aprendizaje (PLE)}

Los entornos personales de aprendizajes o PLE por sus siglas en inglés (Personal Learning Environment), se definen como "el conjunto de herramientas, fuentes de información, conexiones y actividades que cada persona utiliza de forma asidua para aprender"24, es decir, cada uno de nosotros tiene ciertas herramientas que utilizamos para conocer o aprender sobre un tema, y hoy esas herramientas se desenvuelven dentro de círculos virtuales como e-books, blogs, páginas web de noticias, páginas que curan contenidos, videos, etc., que se relacionan con fuentes no virtuales, y todas ellas nos ayudan a aprender y adquirir nuevos conocimientos.

El PLE nos puede servir para integrar las nuevas tecnologías a las clases de manera coherente con los resultados de aprendizaje que queremos obtener, entendiendo que de toda fuente de información podemos aprender y eso, compartirlos con los demás, construyendo conocimiento en conjunto, y no de manera vertical. El PLE se configura con 3 tres procesos cognitivos básicos: leer (en el sentido más amplio de la palabra), reflexionar y compartir ${ }^{25}$.

${ }^{24}$ Linda Castañeda, “Los Entornos Personales de Aprendizajes (PLEs): Una nueva manera de entender el aprendizaje", en Claves para la investigación en innovación y calidad educativas. La integración de las tecnologías de la información y comunicación y la interculturalidad en las aulas, ed. por Rosabel Roig Vila y Massimiliano Fiorucci (Marfil- Roma: Alcoy, TRE Universita degli stud); María J osefina Vidal Ledo et al., "Entornos personales de aprendizaje”, Educación Médica Superior 29, n. ${ }^{\circ} 4$ (2015); César Coll y Anna Engel, "Introducción: los Entornos Personales de Aprendizaje en contextos de educación formal”, Cultura y Educación 26, n. ${ }^{\circ} 4$ (2014): 617-630.

${ }^{25}$ J ordi Adell y Linda Castañeda, "Los Entornos Personales de Aprendizaje (PLE): una nueva manera de entender el aprendizaje", en Claves para la investigación en innovación y calidad educativas. La integración de las tecnologías de la información y comunicación y la interculturalidad en las aulas, eds.. Rosabel Roig Vila y Massimiliano Fiorucci (Marfil- Roma: Alcoy, TRE Universita degli studi); Gisela Martínez Hernández et al., "Los entornos personales de aprendizaje en los procesos de formación formales e informales", Revista Cubana de Educación Médica Superior 30, n.³ (2016): 599-608; César Coll et al., “Los entornos personales de aprendizaje en la educación superior: del diseño al uso", Cultura y Educación 26, 
REVISTA PEDAGOGÍA UNIVERSITARIA Y DIDÁCTICA DEL DERECHO [ISSN 0719-5885]

Unidad de Pedagogía Universitaria y Didáctica del Derecho, Universidad de Chile

$\mathrm{N}^{\circ} 1$, vol. $4,1^{\circ}$ semestre de 2017, 225-253 pp.

Para comprender mejor qué es un PLE, J ordi Adell y Linda Castañeda nos plantean que un PLE tiene tres características:

- Cada alumno se fija sus propios objetivos de aprendizaje.

- No hay evaluaciones, ni títulos; no hay una estructura formal.

- Posibilidad que nos brinda Internet para disponer de un conjunto de herramientas y recursos gratuitos para compartir y aprender a través de ellos

Los mismos autores establecen que un PLE tiene tres partes principales:

- Las herramientas que uno elige para su aprendizaje.

- Los recursos o fuentes de información.

- Personal Learning Network - PLN (Red personal de aprendizaje) que cada uno va construyendo.

Y estos nos proporcionan diferentes herramientas y servicios como:

- Para buscar y acceder a la información.

- Crear editar y publicar información.

- Relaciones con otras personas ${ }^{26}$.

El PLE se relaciona directamente con los "Recursos Educativos Abiertos (REA)", lo que implica que existen materiales de enseñanza abiertos al público, con licencias abiertas que permiten el acceso gratuito, así como el uso, modificación y redistribución por otros sin ninguna restricción o con restricciones limitadas ${ }^{27}$. Estos materiales, sin lugar a dudas enriquecen la construcción del conocimiento, pues permiten el acceso a nuevas informaciones y contenidos.

n. ${ }^{\circ} 4$ (2014): 775-801; Julio Cabero, "El diseño de Entornos Personales de Aprendizaje y la formación de profesores en TIC", Digital Education Review 18, n. ${ }^{\circ 1}$ (2010): 26-37.

26 Adell y Castañeda, "Los Entornos Personales de Aprendizaje (PLE)"

27 UNESCO, Congreso Mundial Sobre Recursos Educativos Abiertos (REA). Declaración de Paris sobre Recursos Educativos Abiertos (REA), 2012. 
En este sentido hay que tener mucho cuidado con el tipo de información a la cual se accede. Es por esto que se hace esencial cuando se estudia el PLE la “agregación, filtrado y curación de los contenidos". Estos conceptos se definen como: "«los sistemas de filtrado son herramientas que ayudan a las personas a encontrar la información más valiosa, de forma que el poco tiempo que se emplee en leer, escuchar o ver sea en los documentos más interesantes y relevantes» (p. 1).

- La agregación la entendemos como la recopilación y presentación de información sobre un mismo tema y contextos determinados que previamente se había filtrado.

- La curación o curación de contenidos (content curation) la entendemos como un concepto más amplio que comprendería también los procesos de filtrado y agregación, comentados anteriormente."28, pero agregándolo valor especial, por el trabajo propio que uno le hace a la información, entregándola nuevamente al medio informático.

Estos conceptos son esenciales, para que ni nosotros ni nuestros estudiantes caigan en lo que se ha llamado infoxicación, que "es la sobrecarga de información o exceso de información provocada por la profusión de contenidos en Internet." 29

Necesariamente, ante esta realidad, y el conocimiento colectivo que se está generando debiésemos empezar a gestionar la información que hay en la Web, para que podamos trabajar con las TIC, en el aula y pasar a las TAC y a las TEP, sin problemas.

28 De Benito et al. "Agregación, filtrado y curación para la actualización docente”.

29 Iñaki Oroz, Qué es la infoxicación y como escapar de ella, 2013 (consultado el 11 de mayo de 2015). http:// papelesdeinteligencia.com/ que-es-la-infoxicacion/ . 


\section{Diseño de actividades en clases de Introducción al Derecho, usando PLE}

Utilizar esta metodología en la sala de clases ha sido esencial para lograr los resultados de aprendizaje declarados, así como alcanzar algunos objetivos en cuanto a las habilidades blandas de nuestros estudiantes, pues le permite ámbitos de autonomía y responsabilidad al estudiante que muchas veces no es posible alcanzarlos de otra manera.

$\mathrm{Si}$ bien es cierto esta actividad se basa en los entornos personales de aprendizajes, ha sido modificado para poder encuadrarlas dentro de las prácticas pedagógicas y requerimientos de nuestro sistema educacional, teniendo en consideración la necesidad de evaluar los objetivos mediante una nota.

En esta parte del trabajo se presentará el contexto que permite realizar estas innovaciones al interior de la Universidad, lo que tributa en los programas de estudio del curso, así como una descripción de la actividad realizada en base al desarrollo de noticias por parte de los estudiantes de Introducción al Derecho I y II.

Contexto: Universidad Católica del Norte y Facultad DE CIENCIAS J URÍDICAS

El proyecto educativo al que adhiere la Universidad Católica del Norte (en adelante UCN) "se hace cargo de los cambios experimentados por la sociedad chilena, la creciente inserción del país en el mundo, los avances científicos y tecnológicos, la globalización del conocimiento, el desarrollo de nuevas formas de comunicación, los modernos enfoques en materia educacional y docente, así 
REVISTA PEDAGOGÍA UNIVERSITARIA Y DIDÁCTICA DEL DERECHO [ISSN 0719-5885]

Unidad de Pedagogía Universitaria y Didáctica del Derecho, Universidad de Chile

$\mathrm{N}^{\circ} 1$, vol. 4, $1^{\circ}$ semestre de 2017, 225-253 pp.

como el impacto que todos estos factores han tenido en el sistema universitario nacional, en general, y en nuestra Casa de Estudios Superiores, en particular"30.

Este marco conceptual, que guía la formación de nuestros estudiantes, tiene como eje fundamental la educación en valores. La enseñanza centrada en el aprendizaje y la formación para la globalización, los que, a partir de la noción de responsabilidad social, articulan una serie de competencias que se pretende desarrollar y potenciar en los estudiantes, para lo cual se ha optado por un enfoque formador basado en competencias, que ha estructurado el currículo de tal manera que apunte al desarrollo integral de éstas en apoyo, además, del uso de las nuevas tecnologías de la información y comunicaciones (TIC) ${ }^{31}$.

En este panorama se inserta la Facultad de Ciencias J urídicas en donde han existido una serie de capacitaciones para integrar las TIC a la sala de clases. En el marco del proyecto Mecesup UCN 0802 el año 2011 se realiza una asistencia técnica que tiene como fin lograr este objetivo ${ }^{32}$. A su vez los profesores deben utilizar la plataforma educa como medio de comunicación oficial con los

\footnotetext{
${ }^{30}$ Universidad Católica Del Norte, Proyecto educativo (consultado 12 de julio de 2015). https:/ / online.ucn.cl/informeAutoevaluacionUCN/?rut_var=11828613\&opcion=1132\&idkey=76 1558298 párrafo 5.9 .

${ }^{31}$ Katherine Becerra y Carolina Salas, "Sistema metodológico integral del aprendizaje del
} Derecho Constitucional a partir de la vivencia: una red de estrategias didácticas", Revista de Pedagogía Universitaria y Didáctica del Derecho 2, n. ${ }^{\circ} 1$ (2015): 68-82; Alperhan Babacan \& Hurriyet Babacan, "A transformative approach to work integrated learning in legal education", Education and Training 57, n. ${ }^{\circ} 2$ (2015): 170-183.

${ }^{32}$ Christian Scheechler, "TIC's y enseñanza del Derecho: una deuda pendiente", en Nuevos desafíos en la enseñanza del derecho en Chile, dir. por Chritian Scheechler (Santiago: Libromar, 2012), 136; Gualberto Buela-Casal y Ángel Castro, "Las tecnologías de la información y la comunicación y la evaluación de la calidad en la educación superior", RUSC Universities and Knowledge Society Journal 6, n.² (2009): 3-8; Claudio Escobedo y Eduardo Arteaga, "Evaluación de los estudiantes y docentes sobre las tecnologías de información y comunicación en los procesos de enseñanza-aprendizaje: una mirada desde la carrera de Sociología de la UC Temuco. Un estudio de caso", Digital Education Review 28 (2015): 102-122. 
estudiantes, para lo cual es necesaria la capacitación, y se ha detectado, en el proceso de levantamiento de perfil de egreso para la configuración de una nueva malla, que el uso de las nuevas tecnologías son una de las competencias que solicitan los empleadores ${ }^{33}$.

\section{Programa De Estudio de LA Asignatura ${ }^{34}$}

La asignatura de Introducción al Derecho está dividida en dos semestres, durante el primer año de la carrera de Derecho.

El primer semestre (Introducción al Derecho I) tiene como resultados de aprendizaje que el estudiante pueda conocer, comprender y manejar en sus relaciones prácticas los conceptos jurídicos fundamentales: Derecho y su razonamiento. Las competencias que quiere desarrollar es la capacidad para detectar el carácter o relevancia jurídica de un problema y el área del Derecho comprometida. Igualmente, el curso forma la competencia lingüística básica para comprender los aspectos jurídicos de un problema, y la capacidad de razonar jurídicamente.

Los contenidos temáticos de la asignatura se basan en tres unidades: I. Razonamiento del Derecho y Teoría de las normas. II. Conceptos Jurídicos Básicos y III. Teoría del Ordenamiento J urídico.

El segundo semestre (Introducción al Derecho II) tiene como resultado de aprendizaje que los estudiantes puedan conocer y comprender el concepto, fundamento, funciones y fines del derecho, las fuentes del Derecho, la ley y la interpretación jurídica. Se mantiene la competencia que se quiere desarrollar agregando la de colaborar en el desarrollo de la capacidad para recopilar información relevante, identificar cuáles son las fuentes básicas de información y sus principios generales de interpretación.

${ }^{33}$ Comité de Curriculum, Facultad de Ciencias J urídicas. Cruce de Tabulación de Encuestas, Sedes Coquimbo y Antofagasta. Proceso de Rediseño Curricular. Universidad Católica del Norte, 2015.

\footnotetext{
${ }^{34}$ Esta Información se encuentra en los programas oficiales de las asignaturas.
} 
REVISTA PEDAGOGÍA UNIVERSITARIA Y DIDÁCTICA DEL DERECHO [ISSN 0719-5885]

Unidad de Pedagogía Universitaria y Didáctica del Derecho, Universidad de Chile

$\mathrm{N}^{\circ} 1$, vol. $4,1^{\circ}$ semestre de 2017, 225-253 pp.

Respecto a los contenidos el curso tiene 5 unidades esenciales: I. Nociones Básicas; II. Fuentes Formales del Derecho; III. Teoría de la Ley; IV. Introducción a la interpretación jurídica; y V. Concepto, fundamento, funciones y fines del Derecho.

\section{DISEÑO DE LA ACTIVIDAD}

La actividad que se desarrolla para lograr que los estudiantes lleguen a los resultados de aprendizaje es la utilización de la noticia, lo que tiene como objetivo central lograr que los estudiantes puedan identificar conflictos de la realidad, aunando TAC y práctica del Derecho, dentro del nivel de competencia inicial que se espera en estudiantes de primer año, esto es que sepan reconocer o identificar un conflicto de relevancia jurídica.

Para lograr esto los estudiantes preparan una ficha de noticias entregada con anterioridad, la cual deben realizar cada semana durante el semestre.

En dicha ficha se identifica fuente, título, fecha, contenido de la noticia y el análisis del estudiante en base a la materia vista en las clases anteriores. Estas noticias deben ser buscadas mediante los curadores de noticias. A los estudiantes se les recomienda utilizar Feedly o Scoop.it, sin perjuicio de que puedan utilizar otros medios.

Feedly es una herramienta para la suscripción de contenidos a través de formatos como RSS, la que permite mantener todas las fuentes de información que se necesiten en un solo sistema, sin tener que visitar cada una de ellas, permitiendo almacenar artículos favoritos y compartir contenidos en las redes ${ }^{35}$.

Scoop.it es un agregador de contenidos que "ayuda a crear tópicos donde vamos incluyendo información que es de nuestro interés de manera pública y abierta a todos los que puedan estar interesados" ${ }^{\prime \prime 3}$.

${ }^{35}$ Aleimys Pichs, "Informe sobre lectura y estudio de herramientas para combatir la infoxicación: feedly y bloglovin" Conference paper: Cómo sobrevivir a la infoxicación a través de las herramientas que nos brinda la web. Octubre de 2013.

${ }^{36}$ De Benito et al. "Agregación, filtrado y curación para la actualización docente”. 
Estos curadores de contenidos, permiten al estudiante, poder crear diferentes categorías de noticias que se relacionen con el contenido de la materia y aunar en un solo sitio, diferentes páginas web, que sean capaces de entregar la información que necesitan.

Estas herramientas, en especial Feedly, tienen como ventaja el ahorro de tiempo; mejoras en la productividad (focalización del contenido); muestra solo los contenidos que interesan a modo de revista informativa individual; $y$, organiza los contenidos junto a las fuentes delimitadas como interesantes o relevantes (evita la búsqueda de links de manera repetitiva) ${ }^{37}$.

Con esto se cumple la primera etapa del PLE, en atención a que se pone el foco en el estudiante y su capacidad para encontrar la información adecuada y la elección de las fuentes de información.

Esto permite a los estudiantes que vayan haciendo aplicación directa a la realidad de las materias vistas en clase, logrando crear el vínculo tan necesario entre teoría y práctica, que muchas veces se pierde en el Derecho, haciéndose cargo de su proceso de aprendizaje mediante la elección adecuada de la noticia, luego aplicando la materia, para finalmente realizar un comentario crítico.

Con esta noticia, el estudiante realiza una exposición de la misma, explicándole a sus compañeros cada uno de estos elementos. En este sentido los estudiantes, pueden recibir preguntas, comentarios, críticas a su trabajo y finalmente discutir sobre las temáticas, nunca perdiendo el foco de la aplicación de la materia. En cada clase exponen dos a tres estudiantes en no más de 10 minutos. Con esta exposición el estudiante logra realizar el análisis crítico y compartir sus conclusiones con sus compañeros, lo que le permite enriquecer el trabajo, logrando la relación con el entorno.

Luego este material es recopilado en un portafolio, que es comprendido como una técnica de recopilación, colección y repertorio de evidencias y competencias profesionales que capacitan a una persona para el desarrollo satisfactorio de

\footnotetext{
${ }^{37}$ Pichs, "Informe sobre lectura y estudio de herramientas para combatir la infoxicación", 4.
} 
REVISTA PEDAGOGÍA UNIVERSITARIA Y DIDÁCTICA DEL DERECHO [ISSN 0719-5885]

Unidad de Pedagogía Universitaria y Didáctica del Derecho, Universidad de Chile

$\mathrm{N}^{\circ} 1$, vol. $4,1^{\circ}$ semestre de 2017, 225-253 pp.

estas competencias $^{38}$. En el mismo se reúnen todas las noticias y da cuenta de cómo se va avanzando y afinando en algunas técnicas que son siempre importantes para los abogados: comprensión lectora, uso del lenguaje escrito, relación de ideas, análisis crítico, etc. Lo interesante de esta evaluación, es que lo realmente importante es la trayectoria del estudiante y se incorpora el potencial de aprendizaje ${ }^{39}$.

En el curso de segundo semestre se le agrega una nueva arista al trabajo realizado, pues los estudiantes mejor evaluados en cada clase, suben al foro de la plataforma educ@ su noticia y los compañeros le hacen comentarios a la misma, nuevamente enriqueciendo el debate, con diversos puntos de vista. Lo esencial en los foros electrónicos es que son centros de discusión de temas en particular que concentran opiniones de diversas personas que están espacialmente en distintos lugares y de manera asincrónica ${ }^{40}$, lo que fortalece el aprendizaje colaborativo de los estudiantes, pues produce un proceso consciente y verdaderamente significativo en relación con sus compañeros ${ }^{41}$.

Con la exposición de la noticia, el portafolio y el foro electrónico, se logra alcanzar la última etapa del PLE: compartir. Es esencial que el análisis crítico y los nuevos aprendizajes gatillados en los estudiantes, sean capaces de ser entregados al sistema, para poder crear nuevos entendimientos y nuevos conocimientos, desarrollando el pensamiento crítico de los estudiantes.

${ }^{38}$ Raquel Barragán, "El Portafolio, metodología de evaluación y aprendizaje de cara al nuevo Espacio Europeo de Educación Superior. Una experiencia práctica en la Universidad de Sevilla", Revista Latinoamericana de Tecnología Educativa 4, n. ${ }^{\circledR} 1$ (2005): 122-140; Porfirio Morán Oviedo, “Aproximaciones teórico-metodológicas en torno al uso del portafolio como estrategia de evaluación del alumno en la práctica docente. Experiencia en un curso de Laboratorio de Didáctica en la docencia universitaria”, Perfiles Educativos 32, n. ${ }^{\circ} 129$ (2010): 102-128; Federico Rodríguez y Luis Pedraza, "Fundamentos para la implantación del portafolio académico como otro elemento en la evaluación del residente”, Medicina Interna de México 25, n. 5 (2009):393394.

39 Barragán, "El Portafolio, metodología de evaluación y aprendizaje de cara al nuevo”, 123.

40 Viviana Brito, "El foro electrónico: una herramienta tecnológica para facilitar el aprendizaje colaborativo", Edutec. Revista Electrónica de Tecnología Educativa 17 (2004): 1-12

41 Brito, "El Foro electrónico: una herramienta tecnológica”. 


\section{Conclusiones}

Hoy en día, la realidad en la sala de clases ha cambiado profundamente, las TIC y las TAC han llegado para quedarse, pues son parte de la realidad de la mayoría de nuestros estudiantes, por tanto, no utilizarlas en el Derecho, lo que produce es tensión entre la realidad del estudiante y lo que hace en la universidad.

Por otro lado, el proceso de aprendizaje es de responsabilidad del estudiante y el foco ya no se encuentra colocado en el profesor, quien se vuelve un facilitador del proceso. En este sentido hay que dejar que nuestros estudiantes se hagan parte del mismo, tomando decisiones en cuanto a qué quieren aprender de los diferentes temas, y con esto no se quiere decir que modifiquen los programas del curso, sino que puedan escoger las fuentes de información en el proceso: "La toma de decisiones es, en sí misma, un proceso de aprendizaje. El acto de escoger qué aprender y el significado de la información que se recibe, es visto a través del lente de una realidad cambiante. Una decisión correcta hoy, puede estar equivocada mañana debido a alteraciones en el entorno informativo que afecta la decisión"42. Esto es una de las bases esenciales del conectivismo, en donde el conocimiento es efectivamente construido entre todos, y utilizando herramientas tecnológicas.

Por esto trabajar con PLE se vuelve esencial en un curso que es generador de bases conceptuales para el resto de la carrera de Derecho, pues permiten la integración de las TAC, el conocimiento disciplinar, y la construcción del conocimiento colectivo, tan esencial para los estudiantes, devolviendo lo aprendido al sistema con nuevos matices y miradas.

Si bien es cierto, en la experiencia narrada no se utilizó un PLE con cada una de sus características plenas, sí es importante señalar que se logró que los estudiantes pudiesen buscar fuentes de información autónomas, utilizando la web como herramienta en su proceso de aprendizaje, pudiendo cada uno de ellos construir su red de aprendizaje y colaborar en el proceso de aprendizaje de

\footnotetext{
${ }^{42}$ Siemmens. Ibíd.
} 
sus compañeros, compartiendo sus conocimientos, con lo cual se cumplió con el objetivo planteado al diseñar esta actividad de trabajo.

\section{Referencias Bibliográficas}

Adell, J ordi y Linda Castañeda. "Los Entornos Personales de Aprendizaje (PLE): una nueva manera de entender el aprendizaje". En Claves para la investigación en innovación y calidad educativas. La integración de las tecnologías de la información y comunicación y la interculturalidad en las aulas. Editado por Rosabel Roig Vila y Massimiliano Fiorucci. Marfil- Roma: Alcoy, TRE Universita degli studi. https:// digitum.um.es/xmlui/handle/ 10201/17247

Argudín, Yolanda. Educación basada en competencias: nociones y antecedentes. México: Trillas, 2005.

Cabero, Julio, Julio Barroso y María Carmen Llorente. “El diseño de Entornos Personales de Aprendizaje y la formación de profesores en TIC". Digital Education Review 18, n. ${ }^{\circ}$ (2010): 26-37.

Coll, César, Anna Engel, Alexandra Saz \& Alfonso Bustos. "Los entornos personales de aprendizaje en la educación superior: del diseño al uso". $\begin{array}{lllll}\text { Cultura y } & \text { Educación } & 26, & \text { n. } & \end{array} \quad$ (2014): 775-801. http:// dx.doi.org/ 10.1080/11356405.2014.985935

Babacan, Alperhan \& Hurriyet Babacan. "A transformative approach to work integrated learning in legal education". Education and Training 57, n. ${ }^{\circ} 2$ (2015): 170-183.

Barragán, Raquel. "El Portafolio, metodología de evaluación y aprendizaje de cara al nuevo Espacio Europeo de Educación Superior. Una experiencia 
práctica en la Universidad de Sevilla". Revista Latinoamericana de Tecnología Educativa 4, n. ${ }^{\circ} \quad$ (2005): 122-140. http:// dialnet.unirioja.es/ descarga/ articulo/ 1303745.pdf

Becerra, Katherine y Carolina Salas. "Sistema metodológico integral del aprendizaje del Derecho Constitucional a partir de la vivencia: una red de estrategias didácticas". Revista de Pedagogía Universitaria y $\begin{array}{llllll}\text { Didáctica del } & \text { Derecho } & 2 & & & \end{array}{ }^{\circ}$ (2015): 68-82. http:// www.pedagogiaderecho.uchile.cl/index.php/RPUD/article/view File/36682/38281

Boza, Ángel y Sara Conde. "Web 2.0 en educación superior: formación, actitud, uso, impacto, dificultades y herramientas". Digital Education Review, n. ${ }^{\circ} 28 \quad$ (2015): 45-58. http:// revistes.ub.edu/index.php/der/article/viewFile/ 14423/pdf

Brincones, Isabel. "La incorporación de las TIC al proceso de enseñanza y aprendizaje". Revista de Investigación e Innovación Educativa del Instituto Universitario de Ciencias de la Educación. Universidad Autónoma de Madrid. http:// www.uam.es/servicios/apoyodocencia/ice/tarbiya/tarbiya/39/3 9-01.html.

Brito, Viviana. "El Foro electrónico: una herramienta tecnológica para facilitar el aprendizaje colaborativo". Edutec. Revista Electrónica de Tecnología Educativa 17 (2004): 1-12. http:// www.edutec.es/revista/index.php/ edutece/article/view/532/266

Buela-Casal, Gualberto y Ángel Castro, Las tecnologías de la información y la comunicación y la evaluación de la calidad en la educación superior 
RUSC. Universities and Knowledge Society Journal 6, n. ${ }^{\circ} 2$ (2009): 38. http:// www.redalyc.org/pdf/780/78012947007.pdf

Cabero, J ulio. "Nueva mirada de las TIC aplicadas en la Educación Superior". Revista Digital Andalucía Educativa (2000): 1-14. http:// www.uv.es/asepuma/XVII/611.pdf

Cabero, Julio. "Capacidades tecnológicas de las TICs por los estudiantes". Enseñanza \& Teaching: Revista interuniversitaria de didáctica $n .^{\circ} 24$ (2006): 159-175. https:// dialnet.unirioja.es/ servlet/articulo?codigo $=2332398$

Cabrera Di Piramo, Carolina y Amílcar Davyt. "Relaciones entre modelos de calidad de la educación superior y de políticas de ciencia, tecnología e innovación". Revista Iberoamericana de Educación Superior 8, n. ${ }^{\circ 21}$ (2017): 109-122

Castañeda, Linda. “Los Entornos Personales de Aprendizajes (PLEs): Una nueva manera de entender el aprendizaje". En Claves para la investigación en innovación y calidad educativas. La integración de las tecnologías de la información y comunicación y la interculturalidad en las aulas. Editado por Rosabel Roig Vila y Massimiliano Fiorucci. Marfil- Roma: Alcoy, TRE Universita degli studi.

Castañeda, Linda y J ordi Adell. "El desarrollo profesional docente en los entornos personales de aprendizajes (PLE)". En Entornos personales de aprendizaje: claves para el ecosistema educativo en red. Editado por Linda Castañeda y Jordi Adell. Alicante: Marfil, 2011. https:// digitum.um.es/jspui/bitstream/10201/30427/ 1/CastanedayAde lllibroPLE.pdf 
Castañeda, Linda y J ordi Adell. “Más allá de la tecnología: análisis de los entornos de aprendizaje personales y grupales de estudiantes en una asignatura universitaria”. Cultura y Educación 26, n. ${ }^{\circ} 4$ (2014): 739774. http:// dx.doi.org/ 10.1080/ 11356405.2014.985946

Casquero, Oskar, Ramón Ovelar, J esús Romo y Manuel Benito. “Entornos de aprendizaje personales, educación superior y analítica del aprendizaje: un estudio sobre los efectos de la multiplicidad de servicios en las redes personales de estudiantes universitarios". Cultura y Educación 26, n. ${ }^{\circ} 4$ (2014):

696-738. www.tandfonline.com.ezproxy.puc.cl/doi/abs/10.1080/11356405.2014. $\underline{985945}$

Comité de Curriculum, Facultad de Ciencias Jurídicas. Cruce de Tabulación de Encuestas, Sedes Coquimbo y Antofagasta. Proceso de Rediseño Curricular. Universidad Católica del Norte, 2015.

Coll, César y Anna Engel. "Introducción: los Entornos Personales de Aprendizaje en contextos de educación formal". Cultura y Educación 26, n. ${ }^{\circ} 4$ (2014): 617-630, DOI:10.1080/11356405.2014.985947

De Benito Crosetti, Bárbara, Antònia Darder Mesquida; Alexandra Lizana Carrió, Victoria Marín Juarros; J uan Moreno García y J esús Salinas Ibáñez. "Agregación, filtrado y curación para la actualización docente". Píxel-Bit. Revista de Medios y Educación n. 42 (2013): 157-169.

Escobedo, Claudio y Eduardo Arteaga. "Evaluación de los estudiantes y docentes sobre las Tecnologías de Información y Comunicación en los procesos de enseñanza-aprendizaje: una mirada desde la carrera de Sociología de la UC Temuco. Un estudio de caso". Digital Education Review 28, (2015):

102-122. http://revistes.ub.edu/index.php/der/article/viewFile/ 11928/pdf 
REVISTA PEDAGOGÍA UNIVERSITARIA Y DIDÁCTICA DEL DERECHO [ISSN 0719-5885]

Unidad de Pedagogía Universitaria y Didáctica del Derecho, Universidad de Chile $\mathrm{N}^{\circ}$ 1, vol. 4, $1^{\circ}$ semestre de 2017, 225-253 pp.

Festinger, León. A theory of cognitive dissonance. Evanston. Illinois: Row Peterson, 1957

Garzón Clemente, Rebeca. "Políticas públicas de inclusión de las tecnologías de la información y comunicación en la educación superior mexicana". $\begin{array}{llllllll}\text { Revista de Pedagogía } 35 & \text { y } & 36 & \text { n. } & 97-98 & \text { (2013). }\end{array}$ http:// www.redalyc.org/pdf/659/65935862007.pdf

Granados Romero, J ohn, Raúl López Fernández, Raidell Avello Martínez, Diego Luna Álvarez, Enrique Luna Álvarez y Walter Luna Álvarez. “Las tecnologías de la información y las comunicaciones, las del aprendizaje y del conocimiento y las tecnologías para el empoderamiento y la participación como instrumentos de apoyo al docente de la universidad del siglo XXI". Medisur 12, n. ${ }^{\circ} 1$ (2014): 289-294. http:// www.medisur.sld.cu/index.php/ medisur/article/view/ 2751

Herrero Martínez, Rafaela, Ignacio González López, Verónica Marín. "Formación centrada en competencias estudiantiles en Educación Superior". Revista de Ciencias Sociales 21, n. ${ }^{\circ} 4$ (2015): 461-478. http://www.redalyc.org/jatsRepo/280/28043815002/28043815002_v isor_jats.pdf

León, Ana Patricia, Eduardo Risco y Cristina Alarcón. "Estrategias de aprendizaje en educación superior en un modelo curricular por competencias". Revista de la Educación Superior 43, n. ${ }^{172}$ (2014): 123-144. $\quad$ http://ac.els-cdn.com/S0185276015000552/1-s2.0S0185276015000552-main.pdf? tid=d4a347be-33fb-11e7-b57a$\underline{00000 a a b 0 f 27 \& a c d n a t=1494254415 \quad \text { cb07fe247110f678178411b6aea89 }}$ 2bd 
Martínez Hernández, Gisela, Nidia Nolla Cao, María Vidal Ledo y Lilia María de la Torre Navarro. "Los entornos personales de aprendizaje en los procesos de formación formales e informales". Revista Cubana de Educación Médica Superior 30, n. ${ }^{\circ} 3$ (2016): 599-608

Morán Oviedo, Porfirio. “Aproximaciones teórico-metodológicas en torno al uso del portafolio como estrategia de evaluación del alumno en la práctica docente. Experiencia en un curso de Laboratorio de Didáctica en la docencia universitaria”. Perfiles Educativos 32, n. ${ }^{\circ} 129$ (2010): 102-128.

Nieto Martín, Santiago y María J osé Rodríguez Conde. “Convergencia de resultados en dos diseños de investigación-innovación en enseñanza universitaria a través de las TIC". Revista Española de Pedagogía 65, n. ${ }^{\circ} 236$ (2007):27-48.

López De La Madrid, María. “Usos de las TIC en la educación superior en México. Un caso de estudio". Revista Apertura (2007): 63-81.

Pichs, Aleimys. "Informe sobre lectura y estudio de herramientas para combatir la infoxicación: feedly y bloglovin" Conference paper: Cómo sobrevivir a la infoxicación a través de las herramientas que nos brinda la web. Octubre de 2013, (consultado el 29 de octubre de 2015) http:// www.researchgate.net/profile/Aleimys Pichs_Fernandez/public ation/263966069 como sobrevivir_a la infoxicacion_a travs de la s herramientas que nos brinda la web/links/0c96053c6e3dcee71c 000000.pdf

Prieto, Vicente, Ileana Quiñones, Giselle Ramírez, Zoe Fuentes, Tahimí Labrada, Orlando Pérez y Melba Montero. “Impacto de las tecnologías de la información y las comunicaciones en la educación y nuevos paradigmas del enfoque educativo". Revista Cubana de Educación Médica Superior 25. n. ${ }^{\circledR 1}$ (2011): 95-102. 
Salinas, Jesús. "Innovación docente y uso de las TIC en la enseñanza universitaria". Revista Universidad y Sociedad del Conocimiento 1, n. ${ }^{\circ} 1$ (2004): 1-16.

Scheechler, Christian. "TIC's y enseñanza del Derecho: una deuda pendiente". En Nuevos desafíos en la enseñanza del derecho en Chile. Dirigido por Chritian Scheechler. Santiago: Libromar, 2012.

Scheechler, Christian. "¿El buey y la carreta? Las TIC al servicio d la planificación de la enseñanza del Derecho". En Nuevos Paradigmas e innovaciones en la Enseñanza del Derecho: Actas II Seminario de Enseñanza del Derecho. Coordinado por Katherine Becerra. Santiago: Ediciones J urídicas de Santiago, 2015.

Sepúlveda, María Del Pilar e Ignacio Calderón, "Las TICs y los procesos de enseñanza-aprendizaje: la supremacía de las programaciones, los procesos de enseñanza y las calificaciones ante las demandas de la sociedad del conocimiento". Revista Iberoamericana de Educación 45, n5 (2007): [http:// rieoei.org/2195.htm

Rodríguez, Federico y Luis Pedraza. Fundamentos para la implantación del portafolio académico como otro elemento en la evaluación del residente". Medicina Interna de México 25, n. 55 (2009):393-394. http:// www.medigraphic.com/pdfs/medintmex/mim2009/mim095i.pdf

Ruiz-Velasco Sánchez, Enrique y Claudia Ortega Barba. “Tecnologías de la información y la comunicación para la innovación educativa". Perfiles Educativos 36, n. ${ }^{\circ} 144$ (2014): 214-218 
REVISTA PEDAGOGÍA UNIVERSITARIA Y DIDÁCTICA DEL DERECHO [ISSN 0719-5885] Unidad de Pedagogía Universitaria y Didáctica del Derecho, Universidad de Chile $\mathrm{N}^{\circ}$ 1, vol. 4, $1^{\circ}$ semestre de 2017, 225-253 pp.

UNESCO. Congreso Mundial Sobre Recursos Educativos Abiertos (REA). Declaración de Paris sobre Recursos Educativos Abiertos (REA), 2012.

Vidal Ledo, María J osefina, Gisela Martínez Hernández, Nidia Nolla Cao y María Niurka Vialart Vidal. "Entornos personales de aprendizaje". Educación Médica Superior 29, n. ${ }^{\circ} 4 \quad$ (2015). http:// www.ems.sld.cu/ index.php/ ems/article/ view/ 726/ 314 